
Aim-To assess the ability of the commer-
cially available monoclonal antibody $1 \mathrm{~B} 12$
(BioGenex, San Ramon, USA) to identify
$C$ albicans in formalin fixed, paraffin wax
embedded material (FFPE).
Methods-Broth cultures of 20 strains of
seven Candida species were resuspended
in $4 \%$ agarose blocks, fixed in formalin for
24 hours, and embedded in paraffin wax.
In addition, 16 blocks of FFPE tissue
known to contain periodic acid-Schiff
positive fungal hyphae were examined.
Antigen retrieval involved microwave
treatment of specimens in citrate buffer
(0.01 M; pH 6.5 ) before addition of $1 B 12$
antibody for 24 hours. Bound antibody
was subsequently detected using a bioti-
nylated link antibody and a peroxidase
conjugated streptavidin.
Results-Only $C$ albicans strains were
$1 B 12$ positive in the agarose blocks. All
FFPE tissue blocks were found to contain
$1 B 12$ positive hyphal structures, indicating the presence of $C$ albicans.

\begin{tabular}{|c|c|c|}
\hline Candida isolate & Source & $\begin{array}{l}\text { Staining with } 1 B 12 \\
\text { antibody }\end{array}$ \\
\hline$C$ albicans $\mathrm{Y} 01.554$ & $\mathrm{HIV}^{\star}$ & Positive \\
\hline$C$ albicans 359/97 & $\mathrm{CHC}$ & Positive \\
\hline$C$ albicans $121 / 94$ & $\mathrm{CHC}$ & Positive \\
\hline C tropicalis Y09.60 & $\mathrm{HIV}^{\star}$ & Negative \\
\hline C tropicalis $340 / 94$ & Dry mouth & Negative \\
\hline C tropicalis $723 / 93$ & $\mathrm{CHC}$ & Negative \\
\hline C krusei $\mathrm{Y} 02.48$ & $\mathrm{HIV}^{\star}$ & Negative \\
\hline C krusei 22/94 & Sjögren's syndrome & Negative \\
\hline C krusei SU6 & Unknownt & Negative \\
\hline C glabrata $\mathrm{Y} 33.149$ & $\mathrm{HIV}^{\star}$ & Negative \\
\hline C glabrata $59 / 94$ & $\mathrm{CHC}$ & Negative \\
\hline C glabrata $644 / 94$ & $\mathrm{CHC}$ & Negative \\
\hline C guilliermondii $\mathrm{Y} 02.02$ & $\mathrm{HIV}^{\star}$ & Negative \\
\hline C guilliermondii $657 / 93$ & Sjögren's syndrome & Negative \\
\hline$C$ parapsilosis NCPF 3104 & Laboratory reference strain & Negative \\
\hline C parapsilosis $937 \mathrm{~A}$ & Unknownt & Negative \\
\hline C parapsilosis 190/94 & Coated tongue & Negative \\
\hline C kefyr Y06.01 & $\mathrm{HIV}^{\star}$ & Negative \\
\hline$C$ kefyr $\mathrm{Y} 06.07$ & $\mathrm{HIV}^{\star}$ & Negative \\
\hline$C$ kefyr $93 / 1290$ & HIV $\ddagger$ & Negative \\
\hline
\end{tabular}

^Courtesy of Pfizer Ltd; †SmithKline Beecham Pharmaceuticals; ¥Guy’s and St Thomas’

Hospital, London. CHC, chronic hyperplastic candidosis. cases of chronic hyperplastic

\section{Immunocytochemical detection of Candida albicans in formalin fixed, paraffin embedded material}

\author{
David W Williams, Helena S Jones, Russ T Allison, A John C Potts, Michael A O Lewis
}

candidosis will help to clarify the role of individ will help to clarify the role of individual Candida spp in this important form of oral candidosis. (F Clin Pathol 1998;51:857-859)

Keywords: Candida albicans; immunocytochemistry; chronic hyperplastic candidosis

Mycological confirmation of the presence of oral candidosis generally involves sampling of the mucosal surface for the culture and subsequent identification of Candida spp. However, chronic hyperplastic candidosis (CHC) differs from the other forms of oral candidosis in that Candida spp invade the superficial layers of the epithelium. As a result the organisms are often only recovered in small numbers or not at all following sampling, a feature that makes it difficult to diagnose CHC on the basis of candidal culture alone. Therefore, biopsy of $\mathrm{CHC}$ lesional tissue and microscopic examination for the presence of structures consistent with fungal hyphae is essential for diagnosis.

Human soft tissue biopsy material is routinely preserved by fixation in formalin and subsequent paraffin wax embedding. Staining with periodic acid-Schiff (PAS) or methenamine-silver stains can detect hyphal structures consistent with Candida spp in fixed tissue sections. However, these stains do not permit identification of individual candida species. The association of $\mathrm{CHC}$ with the

\begin{tabular}{lll} 
Tissue & Origin of tissue & $\begin{array}{l}\text { Staining with } \\
\text { 1B12 antibody }\end{array}$ \\
\hline 1 & CHC & Positive \\
2 & CHC & Positive \\
3 & CHC & Positive \\
4 & CHC & Positive \\
5 & CHC & Positive \\
6 & CHC & Positive \\
7 & CHC & Positive \\
8 & CHC & Positive \\
9 & CHC & Positive \\
10 & CHC & Positive \\
11 & CHC & Positive \\
12 & CHC & Positive \\
13 & Fibroepithelial polyp & Positive \\
14 & Fibroepithelial polyp & Positive \\
15 & CHC & Positive \\
Biomen control & Kidney (HIV patient) & Positive \\
& & \\
\hline
\end{tabular}

CHC, chronic hyperplastic candidosis. 
development of squamous cell carcinoma, ${ }^{1}$ the reported differences in the ability of Candida spp to generate carcinogenic metabolites, ${ }^{2}$ and the variable sensitivity of Candida spp to antifungal agents makes identification of the infecting species important. Previous studies

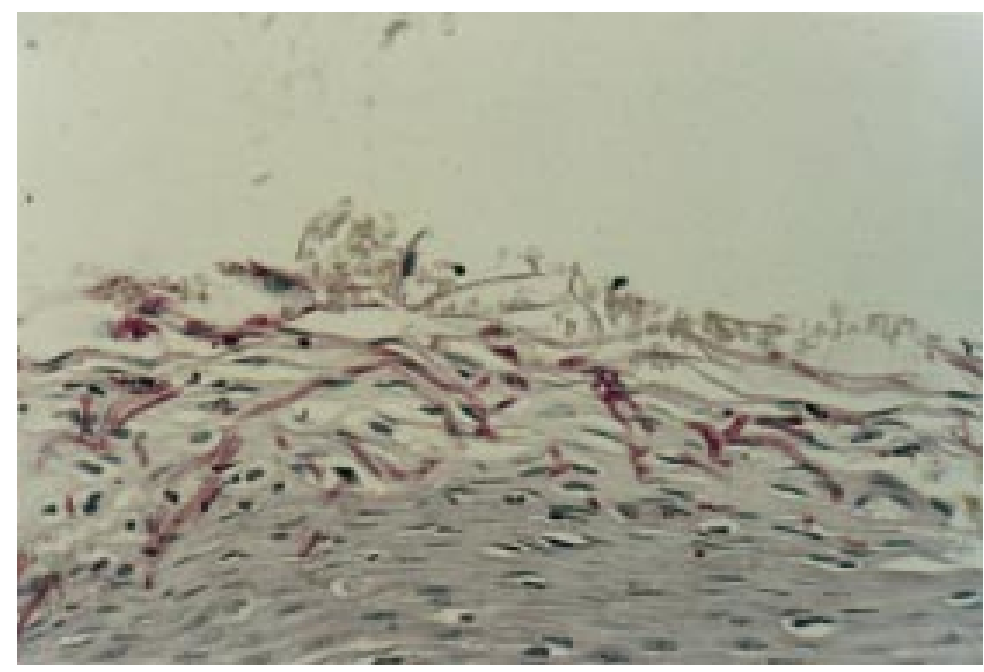

Figure 1 Periodic acid-Schiff staining of oral mucosa from a case of chronic hyperplastic candidosis, showing hyphal structures penetrating epithelium $(\times 111)$.
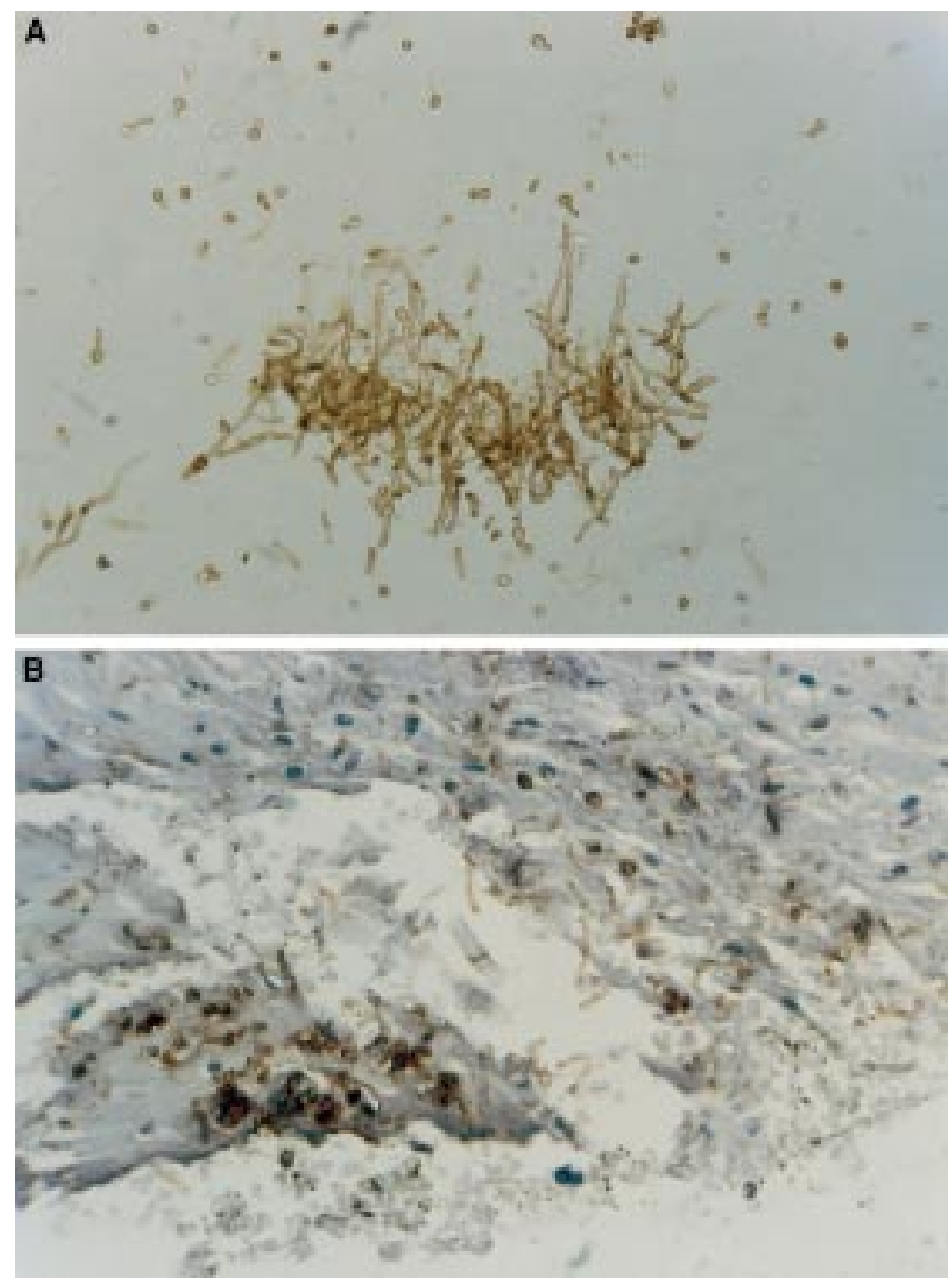

Figure 2 (A) Positive 1 B12 antibody staining of Candida albicans in agarose block section. (B) Tissue section from case of chronic hyperplastic candidosis ( $\times 111)$. have identified Candida spp in CHC biopsies using a polymerase chain reaction (PCR) specific for candidal ribosomal DNA sequences. ${ }^{3}$ However, at present the use of PCR based approaches may not be practical in many routine clinical laboratories. Immunohistological identification of Candida spp has been hindered by the absence of species specific antibodies that are applicable to formalin fixed material. Recently, a mouse monoclonal antibody (1B12) against the high molecular weight mannoproteins of $C$ albicans has become commercially available (BioGenex; distributed in the United Kingdom by Biomen Diagnostics). This antibody is reported to be species specific and suitable for use with formalin fixed, paraffin embedded material. ${ }^{4}$ Our aim in this present study was to assess the specificity of monoclonal antibody (Mab) $1 \mathrm{~B} 12$ and use it to confirm the identity of hyphae in biopsy material from $\mathrm{CHC}$ mucosa.

\section{Methods}

CANDIDAL STRAINS

Twenty isolates of Candida comprising seven species were examined (table 1). All strains were initially identified using the API-20C carbohydrate assimilation system (bioMérieux) and the germ tube test. ${ }^{5}$ All isolates were cultured in horse serum for three hours at $37^{\circ} \mathrm{C}$ to stimulate hyphal growth, before embedding in $0.4 \%$ molten agarose. After solidifying, the embedded agarose material was treated in the same manner as test tissue.

FORMALIN FIXED, PARAFFIN EMBEDDED TISSUE Fifteen mucosal biopsy specimens from separate patients (table 2) were originally fixed in $10 \%$ formal saline for 24 hours before processing through graded alcohol and xylene and embedding in paraffin wax. All tissues were shown by PAS stain to have fungal hyphae penetrating the stratum corneum of the epithelial layer (fig 1) and 13 had the histological features characteristic of CHC. Positive control material was sections of $C$ albicans infected kidney tissue (Biomen Diagnostics).

\section{IMMUNOCYTOCHEMISTRY}

Serial sections $(5 \mu \mathrm{m})$ were cut from formalin fixed, paraffin embedded tissue blocks and placed on SuperFrost microscope slides (Merck). The slides were kept for 24 hours at room temperature before further treatment. Sections were initially equilibrated in citrate buffer (0.01 M; pH 6.5) before microwave treatment (specimens heated at maximum power $(650 \mathrm{~W})$ to boiling and then allowed to simmer for 30 minutes at $455 \mathrm{~W}$ ). Following microwave treatment, the immersed slides were allowed to cool to room temperature. All subsequent staining procedures were performed at room temperature in a humid box to prevent drying of sections. Sections were covered with $3 \%$ hydrogen peroxide in phosphate buffered saline (PBS; pH 7.2;0.01 M; 10 minutes) to block endogenous peroxidase activity, washed in PBS $(3 \times 2$ minutes $)$ and overlaid with goat serum (Biomen Diagnostics) for 10 minutes to block non-specific protein 
binding sites on the section. Excess serum was tipped off and supersensitive Mab 1B12 (Biomen Diagnostics) applied to the wet section. After overnight incubation the sections were washed in PBS ( $3 \times 2$ minutes) and overlaid with biotin labelled goat anti-mouse/rabbit immunoglobulin (20-fold diluted in PBS; Biomen Diagnostics) for 20 minutes. Sections were washed in PBS $(3 \times 2$ minutes $)$ and then overlaid with peroxidase labelled streptavidin (20-fold diluted in PBS; Biomen Diagnostics) for 20 minutes, washed in PBS $(3 \times 2$ minutes), and covered with freshly prepared diaminobenzidine reagent (Sigma; 5 minutes). Sections were then washed in tap water for 2 minutes and overlaid with copper sulphate solution $(0.5 \% \mathrm{wt} / \mathrm{vol}$ in saline; 5 minutes), washed in water, and counterstained with Meyer's haematoxylin for 30 seconds. After washing in tap water, sections were dehydrated in alcohol, cleared in xylene, and mounted with Canada balsam (Fisons).

\section{Results}

Of the 20 isolates of Candida embedded in agarose, positive staining was evident only with those previously identified as $C$ albicans (table 1 ; fig 2A). Positive staining with 1 B12 antibody occurred in all 16 tissue sections where PAS positive hyphae had previously been detected (table 2; fig 2B).

\section{Discussion}

In recent years, it has become evident that Candida spp and strains vary in their production of putative virulence factors, pathogenicity in animal systems, and antifungal susceptibility. Candida spp are frequently associated with superficial infection of mucosal membranes, although fatal systemic infection in debilitated individuals can also occur. Chronic hyperplastic candidosis is a particularly important form of oral candidosis as it is associated with the development of epithelial dysplasia ${ }^{6}$ and intraoral squamous cell carcinoma. Sampling methods for culture of Candida spp are limited to collection from the tissue surface and do not permit the recovery of organisms from within the lesions. Previous studies involving identification of Candida spp by PCR in fixed tissues from $\mathrm{CHC}$ lesions have indicated the presence of $C$ albicans, ${ }^{3}$ although at present this technique is unlikely to be available in the majority of routine diagnostic laboratories.

Our present investigation has shown that monoclonal antibody 1B12 stained all isolates of formalin fixed, paraffin embedded $C$ albicans tested, while cross reactivity with other Candida spp was not evident. Subsequent staining of oral mucosal tissue from CHC and fibroepithelial polyps indicated the presence of $C$ albicans in all specimens examined. The ability to identify candida organisms penetrating the lesional tissue in cases of CHC provides an opportunity to further our knowledge on the role of individual Candida spp in this important form of oral candidosis. It would, however, be of interest to stain these tissues with antibodies specific for other Candida spp in an effort to identify mixed infection. However, to date suitable commercial antibodies do not appear to be available. We thank Mrs K Allsopp for her assistance in histopathology.
DWW was financially supported by the Wales Office of Research and Development for Health and Social Care.

1 Bánóczy J. Follow-up studies in oral leukoplakia. $f$ Maxillofac Surg 1977;5:69-75.

2 Krogh, P. The role of yeast in oral cancer by means of endogenous nitrosation. Acta Odontol Scand 1990;48:85-8.

3 Williams DW, Wilson MJ, Lewis MAO, et al. Identification of Candida species in formalin fixed, paraffin wax embedd Pathol: Mol Pathol 1996;49:M23-8.

4 Monteagudo C, Marcilla A, Mormeneo S, et al. Specific immunohistochemical identification of Candida albicans in paraffin-embedded tissue with a new monoclonal antibody (1B12). Am f Clin Pathol 1995;103:130-5.

5 Taschdjian CL, Reiss F, Kozinn PJ. Experimental vaginal candidiasis in mice; its implications for superficial candidiasis in humans. F Invest Dermatol 1960;34:89-94.

6 Barrett AW, Kingsmill VJ, Speight PM. The frequency of fungal infection in biopsies of oral mucosal lesions. Oral Diseases 1998;4:26-31. 